\title{
Fourth order elliptic boundary value problem with nonlinear term decaying at the origin
}

\author{
Tacksun Jung ${ }^{1}$ and Q-Heung Choi ${ }^{*}$
}

\section{"Correspondence:}

qheung@inha.ac.kr

${ }^{2}$ Department of Mathematics

Education, Inha University, Incheon,

402-751, Korea

Full list of author information is

available at the end of the article

\begin{abstract}
We consider the number of the weak solutions for some fourth order elliptic boundary value problem with bounded nonlinear term decaying at the origin. We get a theorem, which shows the existence of the bounded solution for this problem. We obtain this result by approaching the variational method and using the generalized mountain pass theorem for the fourth order elliptic problem with bounded nonlinear term.
\end{abstract}

MSC: $35 J 30 ; 35 J 40$

Keywords: fourth order elliptic boundary value problem; nonlinear term decaying at the origin; bounded nonlinear term; variational method; generalized mountain pass theorem; $(P S)_{c}$ condition

\section{Introduction}

Let $\Omega$ be a bounded domain in $R^{n}$ with smooth boundary $\partial \Omega$. Let $c \in R^{1}$ and $g: \bar{\Omega} \times R \rightarrow R$ be a $C^{1}$ function. In this paper, we consider the number of the weak solutions for the following fourth order elliptic problem with the Dirichlet boundary condition

$$
\begin{aligned}
& \Delta^{2} u+c \Delta u=g(x, u(x)) \quad \text { in } \Omega, \\
& u=0, \quad \Delta u=0 \quad \text { on } \partial \Omega .
\end{aligned}
$$

We assume that $g \in C^{1}(\bar{\Omega} \times R, R)$ satisfies the following:

(g1) $g \in C^{1}(\bar{\Omega} \times R, R)$,

(g2) $g(x, 0)=0, g(x, \xi)=o(|\xi|)$ uniformly with respect to $x \in \bar{\Omega}$,

(g3) there exists $C>0$ such that $|g(x, \xi)|<C \forall(x, \xi) \in \bar{\Omega} \times R$.

The eigenvalue problem

$$
\begin{aligned}
& \Delta u+\lambda u=0 \quad \text { in } \Omega, \\
& u=0 \quad \text { on } \partial \Omega
\end{aligned}
$$

has infinitely many eigenvalues $\lambda_{j}, j \geq 1$, which is repeated as often as its multiplicity, and the corresponding eigenfunctions $\phi_{j}, j \geq 1$ suitably normalized with respect to $L^{2}(\Omega)$ inner product. The eigenvalue problem

$$
\begin{aligned}
& \Delta^{2} u+c \Delta u=\Lambda u \quad \text { in } \Omega, \\
& u=0, \quad \Delta u=0 \quad \text { on } \partial \Omega,
\end{aligned}
$$

( 2013 Jung and Choi; licensee Springer. This is an Open Access article distributed under the terms of the Creative Commons Attribution License (http://creativecommons.org/licenses/by/2.0), which permits unrestricted use, distribution, and reproduction in any medium, provided the original work is properly cited. 
has also infinitely many eigenvalues $\Lambda_{j}=\lambda_{j}\left(\lambda_{j}-c\right), j \geq 1$ and corresponding eigenfunctions $\phi_{j}, j \geq 1$. We note that

$$
\Lambda_{1}<\Lambda_{2} \leq \Lambda_{3} \leq \cdots, \quad \Lambda_{j} \rightarrow+\infty
$$

Furthermore, we assume that $c \in R^{1}$ satisfies $\lambda_{j}<c<\lambda_{j+1}$.

Jung and Choi [1] proved that (1.1) has at least one nontrivial solution, when $c<\lambda_{1}$ and $g$ satisfies the condition (g1), (g2) and additional conditions

(g3)' there exists $\xi \geq 0$ such that $p(x, \xi) \leq 0 \forall x \in \bar{\Omega}$,

(g4) ${ }^{\prime}$ there exist a constant $r>0$ and an element $e \in H$ such that $\|e\|=r, e<\xi$ and $\frac{1}{2} r^{2}-$ $\int_{\Omega} P(x, e)<0$

by reducing problem (1.1) to the problem with bounded nonlinear term and then applying the maximum principle for the elliptic operator $-\Delta$ and $-\Delta-c$ two times and the mountain pass theorem in the critical point theory. Jung and Choi [2] showed the existence of at least two solutions, one of which is a bounded solution and a large norm solution of (1.1), when $g(u)$ is polynomial growth or exponential growth nonlinear term. The authors proved these results by the variational method and the mountain pass theorem. For the constant coefficient semilinear case Choi and Jung [3] showed that the problem

$$
\begin{aligned}
& \Delta^{2} u+c \Delta u=b u^{+}+s \quad \text { in } \Omega, \\
& u=0, \quad \Delta u=0 \quad \text { on } \partial \Omega,
\end{aligned}
$$

has at least two nontrivial solutions, when $c<\lambda_{1}, \Lambda_{1}<b<\Lambda_{2}$ and $s<0$ or when $\lambda_{1}<c<\lambda_{2}$, $b<\Lambda_{1}$ and $s>0$. The authors obtained these results by using the variational reduction method. The authors [4] also proved that when $c<\lambda_{1}, \Lambda_{1}<b<\Lambda_{2}$ and $s<0$, (1.2) has at least three nontrivial solutions by using the degree theory. Tarantello [5] also studied the problem

$$
\begin{aligned}
& \Delta^{2} u+c \Delta u=b\left((u+1)^{+}-1\right) \quad \text { in } \Omega, \\
& u=0, \quad \Delta u=0 \quad \text { on } \partial \Omega .
\end{aligned}
$$

She showed that if $c<\lambda_{1}$ and $b \geq \Lambda_{1}$, then (1.3) has a negative solution. She obtained this result by the degree theory. Micheletti and Pistoia [6] also proved that if $c<\lambda_{1}$ and $b \geq \Lambda_{2}$, then (1.3) has at least three solutions by the variational linking theorem and LeraySchauder degree theory.

In this paper, we are trying to find weak solutions of (1.1), that is,

$$
\int_{\Omega}\left[\Delta^{2} u \cdot v+c \Delta u \cdot v-g(x, u) v\right] d x=0, \quad \forall v \in H
$$

where $H$ is introduced in Section 2.

We consider the associated functional of (1.1)

$$
I(u)=\int_{\Omega}\left[\frac{1}{2}|\Delta u|^{2}-\frac{c}{2}|\nabla u|^{2}-G(x, u)\right] d x,
$$

where $G(x, s)=\int_{0}^{s} g(x, \tau) d \tau$. By (g1), $I$ is well defined. 
Our main result is the following.

Theorem 1.1 Assume that $\lambda_{j}<c<\lambda_{j+1}, j \geq 1$, and $g$ satisfies the conditions (g1)-(g3). Then (1.1) has at least one bounded weak solution.

We prove Theorem 1.1 by approaching the variational method and using the mountain pass theorem for the reduced fourth order elliptic problem with bounded nonlinear term. The outline of the proof of Theorem 1.1 is as follows: In Section 2, we prove that functional $I(u) \in C^{1}$ and the functional $I$ satisfies the Palais-Smale condition. In Section 3, we show that the functional $I$ satisfies the generalized mountain pass theorem, and so, prove that $I$ has at least one nontrival critical point, from which we prove Theorem 1.1.

\section{Variational approach}

Let $L^{2}(\Omega)$ be a square integrable function space defined on $\Omega$. Any element $u$ in $L^{2}(\Omega)$ can be written as

$$
u=\sum h_{k} \phi_{k} \quad \text { with } \sum h_{k}^{2}<\infty
$$

We define a subspace $H$ of $L^{2}(\Omega)$ as follows

$$
H=\left\{u \in L^{2}(\Omega)\left|\sum\right| \Lambda_{k} \mid h_{k}^{2}<\infty\right\}
$$

Then this is a complete normed space with a norm

$$
\|u\|=\left[\sum\left|\Lambda_{k}\right| h_{k}^{2}\right]^{\frac{1}{2}}
$$

Since $\lambda_{k} \rightarrow+\infty$ and $c$ is fixed, we have $\Lambda_{k} \rightarrow \infty$ and

(i) $\Delta^{2} u+c \Delta u \in H$ implies $u \in H$,

(ii) $\|u\| \geq C\|u\|_{L^{2}(\Omega)}$ for some $C>0$,

(iii) $\|u\|_{L^{2}(\Omega)}=0$ if and only if $\|u\|=0$,

which is proved in [7].

Let

$$
\begin{aligned}
& H_{+}=\left\{u \in H \mid h_{k}=0 \text { if } \Lambda_{k}<0\right\}, \\
& H_{-}=\left\{u \in H \mid h_{k}=0 \text { if } \Lambda_{k}>0\right\} .
\end{aligned}
$$

Then $H=H_{-} \oplus H_{+}$, for $u \in H, u=u^{-}+u^{+} \in H_{-} \oplus H_{+}$. Let $P_{+}$be the orthogonal projection from $H$ onto $H_{+}$and $P_{-}$be the orthogonal projection from $H$ onto $H_{-}$. We can write $P_{+} u=u^{+}, P_{-} u=u^{-}$, for $u \in H$.

By the following Lemma 2.1, the weak solutions of (1.1) coincide with the critical points of the associated functional $I(u)$.

Lemma 2.1 Assume that $\lambda_{j}<c<\lambda_{j+1}, j \geq 1$, and $g$ satisfies the conditions (g1)-(g3). Then $I(u)$ is continuous, and Fréchet differentiable in $H$ with Fréchet derivative

$$
I^{\prime}(u) h=\int_{\Omega}[\Delta u \cdot \Delta h-c \nabla u \cdot \nabla h-g(x, u) h] d x .
$$


If we set

$$
F(u)=\frac{1}{2} \int_{\Omega} G(x, u) d x
$$

then $F^{\prime}(u)$ is continuous with respect to weak convergence, $F^{\prime}(u)$ is compact and

$$
F^{\prime}(u) h=\int_{\Omega} g(x, u) h d x \quad \text { for all } h \in H
$$

this implies that $I \in C^{1}(H, R)$ and $F(u)$ is weakly continuous.

The proof of Lemma 2.1 has the similar process to that of the proof in Appendix B in [8]. Now, we shall show that $I(u)$ satisfies the Palais-Smale condition.

Lemma 2.2 Assume that $\lambda_{j}<c<\lambda_{j+1}, j \geq 1$, and $g$ satisfies the conditions (g1)-(g3). Then the functional I satisfies the Palais-Smale condition: Any sequence $\left(u_{m}\right)$ in $H$, for which $\left|I\left(u_{m}\right)\right| \leq M$ and $I^{\prime}\left(u_{m}\right) \rightarrow 0$ as $m \rightarrow \infty$, possesses a convergent subsequence.

Proof Let us choose $u \in H$. By $g \in C^{1}$ and (g1), $G(x, u)$ is bounded. Then we have

$$
\begin{aligned}
I(u) & =\int_{\Omega}\left[\frac{1}{2}|\Delta u|^{2}-\frac{c}{2}|\nabla u|^{2}-G(x, u)\right] d x \\
& \geq \frac{1}{2}\left\{\lambda_{1}\left(\lambda_{1}-c\right)\right\}\|u\|_{L^{2}(\Omega)}^{2}-\int_{\Omega} G(x, u) d x .
\end{aligned}
$$

Since $u$ is bounded and $\int_{\Omega} G(x, u) d x$ is bounded, $I(u)$ is bounded from below. Thus, $I$ satisfies the $(P S)$ condition.

\section{Proof of Theorem 1.1}

Now, we recall the generalized mountain pass theorem (cf. Theorem 5.3 in [8]).

Let

$$
\begin{aligned}
& B_{r}=\{u \in H \mid\|u\| \leq r\}, \\
& \partial B_{r}=\{u \in H \mid\|u\|=r\} .
\end{aligned}
$$

Theorem 3.1 (Generalized mountain pass theorem) Let $H$ be a real Banach space with $H=V \oplus X$, where $V \neq\{0\}$ and is finite-dimensional. Suppose that $I \in C^{1}(H, R)$ satisfies $(P S)$ condition, and

(i) there are constants $\rho, \alpha>0$ and a bounded neighborhood $B_{\rho}$ of 0 such that

$$
\left.I\right|_{\partial B_{\rho} \cap X} \geq \alpha \text {, and }
$$

(ii) there is an $e \in \partial B_{1} \cap X$ and $R>\rho$ such that if $Q=\left(\bar{B}_{R} \cap V\right) \oplus\{r e \mid 0<r<R\}$, then $\left.I\right|_{\partial Q} \leq 0$.

Then I possesses a critical value $b \geq \alpha$. Moreover, $b$ can be characterized as

$$
b=\inf _{\gamma \in \Gamma} \max _{u \in Q} I(\gamma(u))
$$


where

$$
\Gamma=\{\gamma \in C(\bar{Q}, H) \mid \gamma=\text { id on } \partial Q\}
$$

We shall show that the functional I satisfies the generalized mountain pass geometrical assumptions.

Let $H_{j}=\operatorname{span}\left\{\phi_{1}, \ldots, \phi_{j}\right\}$. Then $H_{j}$ is a subspace of $H$ such that

$$
H=\bigoplus_{j \in N} H_{j} \quad \text { and } \quad H=H_{j} \oplus H_{j}^{\perp} .
$$

Let

$$
Q=\left(\bar{B}_{R} \cap H_{j}\right) \oplus\left\{r e \mid e \in \partial B_{1} \cap H_{j}^{\perp}, 0<r<R\right\} .
$$

Lemma 3.1 Assume that $\lambda_{j}<c<\lambda_{j+1}$ and $g$ satisfies (g1)-(g3). Then

(i) there are constants $\rho>0, \alpha>0$ and a bounded neighborhood $B_{\rho}$ of 0 such that

$$
\left.I\right|_{\partial B_{\rho} \cap H_{j}^{\perp}} \geq \alpha \text {, and }
$$

(ii) there is an $e \in \partial B_{1} \cap H_{j}^{\perp}$ and $R>\rho$ such that if $Q=\left(\bar{B}_{R} \cap H_{j}\right) \oplus\{r e \mid 0<r<R\}$, then $\left.I\right|_{\partial Q} \leq 0$, and

(iii) there exists $u_{0} \in H \backslash Q$ such that $\left\|u_{0}\right\|>R$ and $I\left(u_{0}\right) \leq 0$.

Proof (i) Let $u \in H_{j}^{\perp}$. We note that

$$
\text { if } u \in H_{j}^{\perp}, \quad \int_{\Omega}\left(\Delta^{2} u+c \Delta u\right) u d x \geq \lambda_{j+1}\left(\lambda_{j+1}-c\right)\|u\|_{L^{2}(\Omega)}^{2}>0 .
$$

Since $G(x, u(x))$ is bounded, there exists a constant $C>0$ such that $-C \leq G(x, u(x)) \leq C$. Thus, we have

$$
\begin{aligned}
I(u) & =\frac{1}{2}\left\|P_{+} u\right\|^{2}-\frac{1}{2}\left\|P_{-} u\right\|^{2}-\int_{\Omega} G(x, u) \\
& \geq \frac{1}{2}\left\|P_{+} u\right\|^{2}-C
\end{aligned}
$$

for $C>0$. There exist $\rho>o$ and $\alpha>o$ such that if $u \in \partial B_{\rho} \cap H_{j}^{\perp}$, then $I(u) \geq \alpha$.

(ii) Let us choose an element $e \in \partial B_{1} \cap H_{j}^{\perp}$. Let $u \in\left(\bar{B}_{r} \cap H_{j}\right) \oplus\{r e \mid 0<r\}$. Then $u=v+w$, $v \in B_{r} \cap H_{j}, w=r e$. We note that

$$
\text { if } v \in B_{r} \cap H_{j}, \quad \int_{\Omega}\left(\Delta^{2} v+c \Delta v\right) v d x \leq \lambda_{j}\left(\lambda_{j}-c\right)\|v\|_{L^{2}(\Omega)}^{2}<0 .
$$

Thus, we have

$$
\begin{aligned}
I(u) & =\frac{1}{2} r^{2}-\frac{1}{2}\|v\|^{2}-\int_{\Omega} G(x, v+r e) \\
& \leq \frac{1}{2} r^{2}+\frac{1}{2}\left(\lambda_{j}\left(\lambda_{j}-c\right)\right)\|v\|_{L^{2}(\Omega)}^{2}+C
\end{aligned}
$$


for $C>0$. Then there exists $R>0$ such that if $u \in Q=\left(\bar{B}_{R} \cap H_{j}\right) \oplus\{r e \mid 0<r<R\}$, then $\left.I(u)\right|_{\partial Q} \leq 0$, from which we can choose an element $u_{0} \in H \backslash B_{R}$ such that $I\left(u_{0}\right) \leq 0$.

(iii) If we choose $u_{0} \in H \backslash Q$, then by (ii), $I\left(u_{0}\right) \leq 0$.

Proof of Theorem 1.1 We will show that $I(u)$ has a nontrivial critical point by the generalized mountain pass theorem. By Lemma 2.1, $I(u)$ is continuous and Fréchet differentiable in $H$. By Lemma 2.2, the functional $I$ satisfies $(P S)$ condition. We note that $I(0)=0$. By Lemma 3.1, there are constants $\rho>0, \alpha>0$ and a bounded neighborhood $B_{\rho}$ of 0 such that $\left.I\right|_{\partial B_{\rho} \cap H_{j}^{\perp}} \geq \alpha$, and there is an $e \in \partial B_{1} \cap H_{j}^{\perp}$ and $R>\rho$ such that if $Q=\left(\bar{B}_{R} \cap H_{j}\right) \oplus\{r e \mid 0<r<R\}$. Let us set

$$
\Gamma=\{\gamma \in C(\bar{Q}, H) \mid \gamma=i d \text { on } \partial Q\} .
$$

By the generalized mountain pass theorem, $I$ possesses a critical value $b \geq \alpha$. Moreover, $b$ can be characterized as

$$
b=\inf _{\gamma \in \Gamma} \max _{u \in Q} I(\gamma(u)) .
$$

Thus, we prove that $I$ has at least one nontrivial critical point. We denote by $\tilde{u}$ a critical point of $I$ such that $I(\tilde{u})=b$. We claim that $b$ is bounded. In fact, by (iii) of Lemma 3.1, we have

$$
b \leq \max _{0 \leq t \leq 1} I\left(t u_{0}\right),
$$

and by (g3),

$$
\begin{aligned}
I\left(t u_{0}\right) & =t^{2}\left(\frac{1}{2}\left\|P_{+} u_{0}\right\|^{2}-\frac{1}{2}\left\|P_{-} u_{0}\right\|^{2}\right)-\int_{\Omega} G\left(x, t u_{0}\right) d x \\
& \leq t^{2}\left\|u_{0}\right\|^{2}-\int_{\Omega} G\left(x, t u_{0}\right) d x \\
& \leq t^{2}\left\|u_{0}\right\|^{2}+C_{1}=C_{2} t^{2}+C_{2}
\end{aligned}
$$

for some constant $C_{2}>0$. Since $0 \leq t \leq 1, b$ is bounded:

$$
b<\tilde{C} .
$$

We claim that $\tilde{u}$ is bounded. In fact, by contradiction, $\Delta^{2} \tilde{u}+c \Delta \tilde{u}=g(x, \tilde{u})$ and for any $K>0, \max _{\Omega}|\tilde{u}(x)|>K$ imply that

$$
b=I(\tilde{u})=\frac{1}{2}\left(\left\|P_{+} \tilde{u}\right\|^{2}-\left\|P_{-} \tilde{u}\right\|^{2}\right)-\int_{\Omega} G(x, \tilde{u}) d x
$$

is not bounded, which is absurd to the fact that $b=I(\tilde{u})$ is bounded. Thus, $\tilde{u}$ is bounded, so (1.1) has at least one bounded weak solution. Thus, we prove Theorem 1.1. 


\section{Competing interests}

The authors declare that they have no competing interests.

\section{Authors' contributions}

TJ carried out the studies for the existence of weak solutions of the fourth order elliptic boundary value problem, participated in the sequence alignment and drafted the manuscript. QC participated in the sequence alignment and drafted the manuscript.

\section{Author details}

${ }^{1}$ Department of Mathematics, Kunsan National University, Kunsan, 573-701, Korea. ${ }^{2}$ Department of Mathematics Education, Inha University, Incheon, 402-751, Korea.

\section{Acknowledgements}

This work (Tacksun Jung) was supported by the Basic Science Research Program through the National Research Foundation of Korea (NRF) funded by the Ministry of Education, Science and Technology (KRF-2010-0023985).

Received: 24 April 2013 Accepted: 31 July 2013 Published: 13 September 2013

\section{References}

1. Jung, TS, Choi, QH: Nontrivial solution for the biharmonic boundary value problem with some nonlinear term. Korean J. Math. 21(2), 117-124 (2013)

2. Jung, TS, Choi, QH: Nonlinear biharmonic problem with variable coefficient exponential growth term. Korean J. Math. 18(3), 1-12 (2010)

3. Choi, QH, Jung, T: Multiplicity results on nonlinear biharmonic operator. Rocky Mt. J. Math. 29(1), 141-164 (1999)

4. Jung, TS, Choi, QH: Multiplicity results on a nonlinear biharmonic equation. Nonlinear Anal., Theory Methods Appl. 30(8), 5083-5092 (1997)

5. Tarantello, G: A note on a semilinear elliptic problem. Differ. Integral Equ. 5(3), 561-565 (1992)

6. Micheletti, AM, Pistoia, A: Multiplicity results for a fourth-order semilinear elliptic problem. Nonlinear Anal. TMA 31(7), 895-908 (1998)

7. Choi, QH, Jung, T: Multiplicity of solutions and source terms in a fourth order nonlinear elliptic equation. Acta Math. Sci. 19(4), 361-374 (1999)

8. Rabinowitz, PH: Minimax Methods in Critical Point Theory with Applications to Differential Equations. CBMS. Regional Conf. Ser. Math., vol. 65. Am. Math. Soc., Providence (1986)

doi:10.1186/1029-242X-2013-432

Cite this article as: Jung and Choi: Fourth order elliptic boundary value problem with nonlinear term decaying at the origin. Journal of Inequalities and Applications 2013 2013:432.

\section{Submit your manuscript to a SpringerOpen ${ }^{\circ}$ journal and benefit from:}

- Convenient online submission

- Rigorous peer review

Immediate publication on acceptance

Open access: articles freely available online

- High visibility within the field

- Retaining the copyright to your article 